Questions vives

\section{Questions Vives}

Recherches en éducation

$n^{\circ} 21 \mid 2014$

Le travail collectif des enseignants en question(s)

\title{
« Travailler ensemble » en formation à l'enseignement : une obligation productive
}

Étude de cas à la Haute école pédagogique du Valais (Suisse)

"Working together" in teacher education: a productive obligation. A Case Study at the Valais University of Teacher Education (Switzerland)

\section{Danièle Périsset}

\section{(2) OpenEdition}

\section{Journals}

\section{Édition électronique}

URL : http://journals.openedition.org/questionsvives/1511

DOI : 10.4000/questionsvives. 1511

ISSN : $1775-433 X$

\section{Éditeur}

Université Aix-Marseille (AMU)

\section{Édition imprimée}

Date de publication : 15 septembre 2014

ISBN : 978-2-912643-45-2

ISSN : 1635-4079

\section{Référence électronique}

Danièle Périsset, « «Travailler ensemble » en formation à l'enseignement : une obligation productive », Questions Vives [En ligne], $n^{\circ} 21$ | 2014, mis en ligne le 15 septembre 2014, consulté le 21 décembre 2020. URL : http://journals.openedition.org/questionsvives/1511 ; DOI : https://doi.org/10.4000/ questionsvives. 1511

Ce document a été généré automatiquement le 21 décembre 2020.

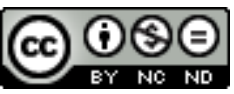

Questions Vives est mis à disposition selon les termes de la licence Creative Commons Attribution Pas d'Utilisation Commerciale - Pas de Modification 4.0 International. 


\section{«Travailler ensemble " en formation à l'enseignement: une obligation productive}

Étude de cas à la Haute école pédagogique du Valais (Suisse)

"Working together" in teacher education: a productive obligation. A Case Study

at the Valais University of Teacher Education (Switzerland)

Danièle Périsset

\section{Faire investir et analyser le «travailler ensemble » en formation initiale à l'enseignement}

1 La recherche dont il est fait état ici a été mise en place dans une visée compréhensive pour documenter empiriquement le processus de construction de la collaboration pédagogique entre stagiaires en début de formation initiale dans une Haute école pédagogique (HEP) de Suisse romande. Les résultats serviront à améliorer en connaissance de cause le dispositif d'alternance et de développement professionnel proposé par l'institut de formation. Autre point à relever par rapport à cette recherche : si le « travailler ensemble » des enseignants au sein des établissements est à présent assez largement documenté, celui des stagiaires en formation initiale l'est beaucoup moins, du moins à notre connaissance. La présente contribution propose d'analyser, le temps d'une étude de cas, la manière dont des étudiants et étudiantes en formation à l'enseignement investissent l'espace collaboratif consubstantiel d'un stage.

\subsection{Travailler ensemble, une caractéristique du métier d'enseignant}

2 La forme même de ce stage accompagné et en duo (deux stagiaires dans la même classe dirigée par un enseignant expérimenté) les contraint à travailler ensemble, caractéristique 
que l'on reconnait aujourd'hui comme étant fondatrice du travail enseignant (Barrère, 2002 ; Marcel, Dupriez, Périsset \& Tardif, 2007 ; Corriveau, Letor, Périsset \& Savoie, 2010), que ce soit spontanément ou que le collectif soit « subi » (Marcel \& Garcia, 2010), comme c'est le cas dans la situation que nous analysons ici. Nous savons aussi que les acteurs n'appliquent jamais telle quelle une décision qui leur est imposée : ils la travaillent, en « interrogent le sens, la praticabilité et légitimité morale et cognitive; ils la situent par rapport à leurs intérêts, à leur expérience et à leur situation; ils la confrontent à ce que leurs collègues pensent et font [...]. » (Lessard, 2010, p. 300).

Pour que travailler ensemble, que ce soit de manière volontaire ou imposée, soit productif, c'est-à-dire utile et efficace, il faut d'une part lui trouver du sens (processus de sensmaking , voir Dupriez, 2007), il faut d'autre part que certaines conditions organisationnelles soient remplies. Letor (2009) en a identifié plusieurs: les moments favorables au cours desquels les acteurs sont disponibles et productifs; les réseaux de collaboration, les acteurs concernés, les compétences mobilisées ; les modalités d'organisation, la prise d'information, l'intensité des rencontres, les facteurs individuels, collectifs et organisationnels; la définition des objets et finalités du travail collaboratif - objets institutionnels, relatifs au travail en classe ou hors de la classe, aux pratiques éducatives en vigueur dans l'établissement ou la classe, de la vie au sein de l'établissement -, les finalités poursuivies par l'équipe enseignante et enfin les indicateurs d'efficacité à disposition de l'équipe et de sa direction et les pistes de régulation à envisager.

On peut faire l'hypothèse que ce qui, dans le domaine du travailler ensemble, est vrai pour les enseignants chevronnés et est décrit par la littérature (voir par ex. Letor, 2009; Corriveau et al., 2010; Marcel et al., 2007), l'est aussi pour les novices en formation professionnelle. On sait pourtant que les novices, catégorie à laquelle appartiennent les enseignants en formation, relèvent d'un genre aux caractéristiques spécifiques (Saujat, 2004). Il faut, simultanément, réaliser un double apprentissage, « "prendre" la classe et apprendre son métier » (Saujat, 2004, p. 99) - engager les élèves dans les apprentissages tout en apprenant soi-même les gestes du métier. Il faut aussi prendre et tenir la classe avant de la faire, pour cela faire appel à toutes sortes de stratégies pour conserver un cadre dans lequel la discipline est contenue alors que faire la classe implique un engagement dans les procédures didactiques.

5 Il est probable que le «travailler ensemble » des stagiaires observés ici aura pour objet ces préoccupations premières: prendre et tenir la classe en plus de répondre correctement aux exigences et prescriptions posées par l'institut de formation.

\subsection{Contexte: des prescriptions pour les stages}

6 Les prescriptions que reçoivent les stagiaires que nous avons observés dans le cadre de cette enquête sont à - au moins - deux niveaux. À un premier niveau, il y a les objectifs de formation propres au stage. En effet, chacun des six stages des trois ans de formation ${ }^{1}$ fait l'objet d'objectifs de développement professionnel à soigner spécifiquement, objectifs qui vont de la sensibilisation (lors du premier stage) à la gestion de l'hétérogénéité, à la responsabilité professionnelle (stages finaux) en passant par la pratique de l'évaluation pédagogique (objectif du stage qui nous intéresse ici, effectué au semestre 2, première année de formation). Pour chacun des stages, sont décrits de manière précise les travaux attendus pour différents cours ou pour les analyses des pratiques; est explicité le rôle attendu des différents protagonistes ${ }^{2}$, la fréquence et la durée des plages d'enseignement 
que les stagiaires doivent organiser et gérer en autonomie. Ces indications sont autant de guides pour l'action. Elles renvoient tant à la dimension collective des tâches à réaliser en mettant en relation les différents professionnels impliqués (Amigues, 2003), qu'à la dimension individuelle, à ce qui est spécifiquement attendu des stagiaires par l'institut de formation ou peut être attendu d'eux par les professionnels chevronnés qui les encadrent.

7 L'autre niveau de prescriptions relatives aux modalités du «travailler ensemble » n'est pas complètement laissé au hasard, même si prescrire le « travailler ensemble » et surtout les processus mobilisés est sans doute plus difficile. De fait, en stage, il faut travailler ensemble parce qu'on y est contraint par le cadre de formation: chaque stagiaire doit enseigner sous le regard d'un enseignant expérimenté désigné par la HEP (le praticienformateur) et entrer en dialogue réflexif avec lui (Périsset \& Buysse, 2008; Buysse, 2009, 2011). Le stagiaire doit aussi pouvoir expliciter le processus évolutif de la construction de ses compétences professionnelles dans l'interaction avec l'autre stagiaire s'il y en a un et avec le superviseur HEP venu en observation et analyse de pratique pendant une demijournée au cours de la dernière semaine de stage. Le travail réflexif et collectif de conscientisation, d'explicitation, de secondarisation de l'expérience est donc cadré par les prescriptions de stage. Les praticiens-formateurs et les superviseurs ont d'ailleurs reçu une formation spécifique à ce propos, une formation à l'accompagnement.

Dans le cadre du stage analysé ici en particulier, il va aussi falloir travailler ensemble parce qu'on va enseigner en "duo", en devant donc se coordonner étroitement, voire collaborer ou coopérer (pour une typologie des ces niveaux, voir Marcel \& Murillo dans ce volume). Les modalités d'interactions permettant de développer une pratique réflexive ont été définies au préalable et sont communiquées aux stagiaires: une démarche de supervision entre pairs est travaillée dans le cadre des rencontres autour du portfolio de développement professionnel. Est également donné aux stagiaires et à leurs praticiensformateurs un canevas d'entretien de formation où, en analyse a posteriori, il leur est demandé de profiter de ces moments institutionnalisés pour mettre en évidence les éléments de cadrage (les faits saillants observés), de recadrage (mise en lien des observations avec les éléments théoriques permettant de comprendre ce qui s'est passé et de réguler; de la situation concrète aux concepts), d'identification des savoirs professionnels investigués (savoirs qui allient intiment expérience et éclairages théoriques adéquats). Cela permet de planifier un plan d'action concret, d'identifier les régulations utiles et de développer en continu une pratique de la réflexion sur et dans l'action (Buysse, 2009).

Dans ce cadre prescrit, il y donc du sens à construire pour soi, par rapport aux prescriptions, entre pairs ou avec le praticien-formateur ou avec le superviseur, en fonction des modalités mises en place d'une part par l'institut de formation mais aussi en fonction de l'interprétation qu'en font les acteurs concernés. L'enjeu, pour les stagiaires, est évidemment d'atteindre les objectifs de formation professionnelle posés puisque la validation du stage dépendra des évaluations posées par le praticien-formateur et le superviseur.

\section{Question de recherche et méthode}

Suite à une enquête menée en 2006 et publiée en 2010 (Périsset, 2010) à propos de l'acculturation au «travailler ensemble» en formation initiale à l'enseignement, nous 
pouvons raisonnablement estimer que, dans le contexte global de la formation à l'enseignement à la HEP du Valais (Suisse) où a lieu la présente enquête, les étudiants et étudiantes s'acculturent tout au long de la formation au fait de «devoir» travailler ensemble à de multiples occasions et y trouvent du bénéfice, voire même du plaisir. Nous nous sommes dès lors demandée comment, concrètement, alors qu'ils ont commencé leur formation depuis un semestre, ils investissent, mettent du sens, s'adaptent et s'approprient l'injonction travaillez ensemble! que leur adresse l'institut de formation, comment ils organisent ce "travailler ensemble» qui leur est imposé, quels objets y sont prioritairement discutés.

11 Un questionnaire investiguant la problématique du « travailler ensemble » spontanément ou parce qu'il s'agit d'une contrainte institutionnelle a été construit à l'aide des propositions de Letor (2009). Les items proposés concernent les participants aux rencontres, le moment de la réunion, le lieu où elle se déroule, les objets principaux qui $\mathrm{y}$ sont discutés ainsi que les décisions prises à l'issue de la réunion.

Ce questionnaire a été remis au printemps 2013 aux 45 étudiantes et étudiants en stage accompagné du second semestre de formation. Il a été clairement spécifié que si la tenue $\mathrm{du}$ «carnet de bord» constitué par l'addition des fiches remplies à l'issue de chaque rencontre vécue au cours du stage était obligatoire et que cette réalisation serait vérifiée lors $\mathrm{du}$ transfert des données sur le questionnaire informatique (obligation institutionnelle afin de permettre à la recherche de se fonder sur un matériau consistant), il n'y aurait aucune évaluation concernant leur contenu ni le nombre de fiches documentées. Lors de la présentation de la recherche, les étudiants se sont montrés curieux de l'expérience et des résultats dont la communication rapide leur a été promise. Lors du dépouillement des données, on a pu constater qu'ils y ont vraiment participé en relevant un maximum de moments de "travail ensemble ", aucun n'ayant rempli son carnet de bord a minima, attitude qui aurait été possible dès lors qu'ils avaient la garantie de ne pas être évalués sur le contenu ni sur la quantité.

Le carnet de bord a donc été renseigné au fur et à mesure des rencontres de 15 minutes au moins (une fiche pour chacune), rencontres formelles ou informelles, spontanées ou agendées, au cours desquelles les stagiaires ont «travaillé ensemble » avec leur collègue de stage, avec leur praticien-formateur ou avec quelqu'un d'autre. De retour en institution, les stagiaires ont introduit leurs données dans un "carnet de bord" informatisé, soit une page web par rencontre. Une analyse statistique descriptive a permis d'établir le profil des rencontres enregistrées. Afin d'affiner les résultats, les variables les plus saillantes pour le présent objet de recherche (à savoir les objets discutés et l'appréciation des rencontres) ont été croisées avec les acteurs présents lors de la rencontre (le co-stagiaire uniquement ou quelqu'un d'autre). Le calcul ${ }^{4}$ a été effectué avec le R-paquet « Survey » ${ }^{5}$. Ce sont les résultats de cette enquête qui sont discutés ici.

\section{Résultats}

\subsection{De fréquentes rencontres}

14 À propos de la fréquence des rencontres : les 45 stagiaires ont rempli 1285 fiches (=n), soit une fiche par rencontre documentée, ce qui donne une moyenne de 28.5 rencontres pour 15 journées complètes et 4 demi-journées de stage réparties sur 4 semaines. Plus d'une 
rencontre par jour a donc eu lieu, ce qui montre que «travailler ensemble » a dépassé le stade de l'injonction et que sa mise en œuvre a été investie.

\subsection{Des rencontres de durées variables}

15 Leur durée : environ un quart des rencontres $(339 ; \mathrm{n}=1285)$ a pris plus d'une heure, le tiers (421) entre 30 et 60 minutes, 525 furent plus brèves (entre 15 et 30 minutes). À noter que les rencontres de moins de 15 minutes n'ont pas été comptabilisées. La majorité des rencontres ont donc été consistantes du point de vue du temps qui leur a été consacré.

\subsection{Les acteurs présents lors des rencontres : les stagiaires entre eux d'abord}

16 Qui était présent ? Les $10 \%$ des rencontres (soit 124) ont été le fait d'un stagiaire seul avec un autre acteur (qui n'était pas son collègue de stage); $1161(\mathrm{n}=1285)$ ont été réalisées par le duo de stagiaires, entre eux ou avec d'autres acteurs du centre scolaire. Plus de la moitié des rencontres a été réalisée avec les praticiens-formateurs $(754 ; n=1285)$, ce qui correspond à la prescription institutionnelle, au mandat que reçoivent les PF et au souci qu'ils ont d'accompagner de manière étroite ces stagiaires en début de formation professionnelle. Il a été en outre relevé que des rencontres ont eu lieu avec d'autres acteurs: ce sont les autres enseignants de l'établissement qui viennent en tête des rencontres effectuées hors praticien-formateur (65), 22 l'ont été avec le co-titulaire ou un remplaçant, 9 stagiaires ont rencontré d'autres enseignants ou agents spécialisés intervenant dans la classe de stage, 10 ont rencontré d'autres stagiaires de la HEP placés dans le même établissement, 3 stagiaires seulement ont rencontré le directeur de l'établissement, 2 ont pu parler avec l'inspecteur scolaire et 2 seulement ont rencontré des parents.

\subsection{Les moments choisis pour les rencontres : de préférence autour des moments d'enseignement}

Les rencontres ont eu lieu à différents moments $(n=1285)$, et notamment :

- 575 rencontres ont eu lieu après la journée de cours, en fin d'après-midi,

- 274 avant les cours,

- 120 pendant la pause de midi,

- 76 en soirée,

- 72 pendant la récréation (qui dure une quinzaine de minutes),

- 47 pendant les heures de classe (soit quand l'autre stagiaire donne un cours, ou quand un intervenant externe prend en charge la classe),

- 19 pendant des jours de congé ou fériés (dimanche, lundi de Pâques) et 10 le mercredi aprèsmidi (congé hebdomadaire des élèves).

\subsection{Les lieux de rencontres : le bâtiment scolaire}

Dans la grande majorité, les rencontres ont eu lieu dans le bâtiment scolaire (1115). Plusieurs stagiaires profitent cependant du trajet pour discuter entre eux (108), certains 
se voient à leur domicile (38), dans un café ou au restaurant (7) ou encore communiquent par téléphone (17).

\subsection{Rencontres majoritairement prévues, souvent aussi spontanées}

19 Les rencontres étaient planifiées dans la majorité des cas $(695 ; \mathrm{n}=1285)$, mais nombreuses (590) ont été les rencontres spontanées, décidées sur le moment.

\subsection{Les objets principalement discutés (seuls 2 objets sur les 12 proposés peuvent être cochés)}

Ils ont été, dans l'ordre d'importance :

- les contenus des cours à donner (555)

- l'organisation matérielle (468)

- les difficultés rencontrées pendant l'enseignement (235)

- les méthodes d'enseignement utilisées (154)

- les réussites constatées pendant l'enseignement (152)

- les élèves en difficulté (138)

- la gestion de la discipline (113)

- divers éléments en lien avec le travail demandé par la HEP pendant le stage (66)

- l'entretien avec le superviseur HEP (63)

- l'élaboration ou l'échange de matériel pédagogique (55)

- l'entretien de supervision avec le praticien-formateur qui a lieu après les cours donnés par le ou les stagiaires (51)

- autre (31)

\subsection{L'utilité perçue de la rencontre ou de la réunion}

À la suite de la rencontre ou de la réunion (2 réponses possibles) :

- dans la moitié des cas (606), une décision a été prise,

- 569 rencontres ont été, pour les stagiaires, l'occasion déclarée d'apprendre consciemment quelque chose,

- 480 ont permis l'échange d'informations ou d'impressions.

Seules 48 rencontres, sur les 1285 que compte l'échantillon, ont été jugées inutiles par les stagiaires.

\subsection{Les objets privilégiés lors des rencontres entre stagiaires}

Suite à la description des caractéristiques des rencontres comptabilisées, la question des objets privilégiés lors des rencontres entre stagiaires ou avec d'autres acteurs - nous avons vu que les praticiens-formateurs étaient ces autres interlocuteurs privilégiés - s'est posée. Y a-t-il des objets qui sont prioritairement discutés entre soi ou avec un autre acteur, ou l'interlocuteur est-il indifférent?

Des différences significatives sont apparues (seuil de significativité: 0.05). Ainsi, ont été significativement davantage discuté avec un autre acteur de l'établissement scolaire (majoritairement avec le praticien-formateur, interlocuteur privilégié des rencontres 
hors pairs), l'organisation matérielle de la journée ou des journées à venir et la préparation des contenus des cours (tableaux 1 et $2^{6}$ ):

Tableau 1 : L'organisation matérielle de la journée ou des journées à venir

\begin{tabular}{|cll|l|l|l|}
\hline & & Non choisi & Choisi & Total \\
\hline & Avec pair de stage & Effectif & 260 & 195 & 455 \\
uniquement & Effectif théorique & 289.3 & 165.7 & 455.0 \\
& Résidu & -29.3 & 29.3 & \\
& & & & \\
& Avec un autre acteur & Effectif & 557 & 273 & 830 \\
& de l'établissement & Effectif théorique & 527.7 & 302.3 & 830.0 \\
& scolaire & Résidu & 29.3 & -29.3 & \\
& & & & \\
Total & & Effectif & 817 & 468 & 1285 \\
& & Effectif théorique & 817.0 & 468.0 & 1285.0 \\
\hline
\end{tabular}

[Résidu: -29.3 / Pearson's X^2: Rao \& Scott adjustment; $F=8.8838, n d f=1, d d f=199, p-$ value $^{7}=$ $0.003236]$

Tableau 2 : La préparation des contenus des cours

\begin{tabular}{|c|c|c|c|c|c|}
\hline & & & Non choisi & Choisi & Total \\
\hline & \multirow{3}{*}{$\begin{array}{l}\text { Avec pair de stage } \\
\text { uniquement }\end{array}$} & Effectif & 192 & 263 & 455 \\
\hline & & Effectif théorique & 258.5 & 196.5 & 455.0 \\
\hline & & Résidu & -66.5 & 66.5 & \\
\hline & \multirow{3}{*}{$\begin{array}{l}\text { Avec un autre acteur } \\
\text { de l'établissement } \\
\text { scolaire }\end{array}$} & Effectif & 538 & 292 & 830 \\
\hline & & Effectif théorique & 471.5 & 358.5 & 830.0 \\
\hline & & Résidu & 66.5 & -66.5 & \\
\hline \multirow[t]{2}{*}{ Total } & & Effectif & 730 & 555 & 1285 \\
\hline & & Effectif théorique & 730.0 & 555.0 & 1285.0 \\
\hline
\end{tabular}

[Résidu $=-66.5 / F=48.3661, n d f=1, d d f=199, p$-value $=4.971$ e-11 $]$

Ont par contre été significativement davantage discuté avec le pair, soit le co-stagiaire hors de la présence d'un tiers et donc hors de la présence du praticien-formateur: les difficultés rencontrées pendant l'enseignement, les réussites constatées pendant l'enseignement, l'entretien de supervision qui a lieu après les cours donnés par le ou les stagiaires (tableaux 3 à 6). 
Tableau 3 : Les difficultés rencontrées pendant l'enseignement

\begin{tabular}{|cll|c|c|c|}
\hline & & Non choisi & Choisi & Total \\
\hline Avec pair de stage & Effectif & 399 & 56 & 455 \\
uniquement & Effectif théorique & 371.8 & 83.2 & 455.0 \\
& Résidu & 27.2 & -27.2 & \\
& & & & \\
& Avec un autre acteur \\
de l'établissement & Effectif & Effectif théorique & 678.2 & 151.8 & 830 \\
& scolaire & Résidu & -27.2 & 27.2 & \\
& & & 1050 & 235 & 1285 \\
Total & & Effectif & 1050.0 & 235.0 & 1285.0 \\
& & Effectif théorique
\end{tabular}

[Résidu $=23.8 / F=7.4877, \mathrm{ndf}=1, \mathrm{ddf}=199, \mathrm{p}$-value $=0.006774]$

Tableau 4 : Les réussites constatées pendant l'enseignement

\begin{tabular}{|c|c|c|c|c|c|}
\hline & & & Non choisi & Choisi & Total \\
\hline & \multirow{3}{*}{$\begin{array}{l}\text { Avec pair de stage } \\
\text { uniquement }\end{array}$} & Effectif & 425 & 30 & 455 \\
\hline & & Effectif théorique & 401.2 & 53.8 & 455.0 \\
\hline & & Résidu & 23.8 & -23.8 & \\
\hline & \multirow{3}{*}{$\begin{array}{l}\text { Avec un autre acteur } \\
\text { de l'établissement } \\
\text { scolaire }\end{array}$} & Effectif & 708 & 122 & 830 \\
\hline & & Effectif théorique & 731.8 & 98.2 & 830.0 \\
\hline & & Résidu & -23.8 & 23.8 & \\
\hline \multirow[t]{2}{*}{ Total } & & Effectif & 1133 & 152 & 1285 \\
\hline & & Effectif théorique & 1133.0 & 152.0 & 1285.0 \\
\hline
\end{tabular}

[Résidu $=23.8 / F=13.1335, \mathrm{ndf}=1, \mathrm{ddf}=199, \mathrm{p}$-value $=0.0003683]$

Tableau 5 : L'entretien de supervision après les cours (avec pair et PF) donnés par l'un ou l'autre stagiaire

\begin{tabular}{|c|c|c|c|c|c|}
\hline & & & Non choisi & Choisi & Total \\
\hline & \multirow{3}{*}{$\begin{array}{l}\text { Avec pair de stage } \\
\text { uniquement }\end{array}$} & Effectif & 454 & 1 & 455 \\
\hline & & Effectif théorique & 436.9 & 18.1 & 455.0 \\
\hline & & Résidu & 17.1 & -17.1 & \\
\hline & \multirow{3}{*}{$\begin{array}{l}\text { Avec un autre acteur } \\
\text { de l'établissement } \\
\text { scolaire }\end{array}$} & Effectif & 780 & 50 & 830 \\
\hline & & Effectif théorique & 797.1 & 32.9 & 830.0 \\
\hline & & Résidu & -17.1 & 17.1 & \\
\hline \multirow[t]{2}{*}{ Total } & & Effectif & 1234 & 51 & 1285 \\
\hline & & Effectif théorique & 1234.0 & 51.0 & 1285.0 \\
\hline
\end{tabular}

[Résidu : 17.1 / F = 21.6448, ndf = 1, ddf = 199, p-value = 5.977e-06] 
Tableau 6 : L'entretien de supervision avec le superviseur HEP

\begin{tabular}{|lll|l|l|l|}
\hline & & Non choisi & Choisi & Total \\
\hline \multirow{2}{*}{$\begin{array}{llll}\text { Avec pair de stage } \\
\text { uniquement }\end{array}$} & Effectif & 446 & 9 & 455 \\
& Effectif théorique & 436.6 & 18.4 & 455.0 \\
& Résidu & 9.4 & -9.4 & \\
Avec un autre acteur & Effectif & 787 & 43 & 830 \\
de l'établissement & Effectif théorique & 796.4 & 33.6 & 830.0 \\
& scolaire & Résidu & -9.4 & 9.4 & \\
& & & & \\
Total & Effectif & 1233 & 52 & 1285 \\
& & Effectif théorique & 1233.0 & 52.0 & 1285.0 \\
\hline
\end{tabular}

[Résidu : 9.4 / F = 10.7756, ndf = 1, ddf = 199, p-value = 0.001215]

Les variables relatives aux objets discutés, à la prise de décision, à avoir appris quelque chose et au fait que la réunion était uniquement un échange d'informations ou d'impressions ont également été analysées plus finement. Il s'avère que les stagiaires ont estimé avoir appris quelque chose significativement plus souvent lorsqu'ils ont rencontré leur co-stagiaire. Les rencontres avec les praticiens-formateurs ont, par contre, été perçues significativement comme étant d'abord des réunions d'échange d'informations ou d'impressions uniquement (tableaux 7 et 8) :

Tableau 7 : Suite à la rencontre, j'ai appris quelque chose

\begin{tabular}{|cll|l|l|l|}
\hline & & & Non choisi & Choisi & Total \\
\hline \multirow{4}{*}{$\begin{array}{c}\text { Avec pair de stage } \\
\text { uniquement }\end{array}$} & Effectif & 350 & 105 & 455 \\
& & Effectif théorique & 253.5 & 201.5 & 455.0 \\
& & Résidu & 96.5 & -96.5 & \\
& & & & \\
& Avec un autre acteur de & Effectif & 366 & 464 & 830 \\
& l'établissement scolaire & Effectif théorique & 462.5 & 367.5 & 830.0 \\
& & Résidu & -96.5 & 96.5 & \\
Total & & Effectif & 716 & 569 & 1285 \\
& & Effectif théorique & 716.0 & 569.0 & 1285.0 \\
\hline
\end{tabular}

[Résidu = 96.5 / F = 124.5339, ndf = 1, ddf = 199, p-value $<2.2 \mathrm{e}-16$ ] 
Tableau 8 : C'était une réunion d'échange d'informations ou d'impressions uniquement

\begin{tabular}{|ll|l|l|l|}
\hline & & Non choisi & Choisi & Total \\
\hline Avec pair de stage & Effectif & 196 & 259 & 455 \\
uniquement & Effectif théorique & 285.0 & 170.0 & 455.0 \\
& Résidu & -89.0 & 89.0 & \\
& & & & \\
Avec un autre acteur de & Effectif & 609 & 221 & 830 \\
l'établissement scolaire & Effectif théorique & 520.0 & 310.0 & 830.0 \\
& Résidu & 89.0 & -89.0 & \\
& & & & \\
& Effectif & 805 & 480 & 1285 \\
& Effectif théorique & 805.0 & 480.0 & 1285.0 \\
\hline
\end{tabular}

[Résidu = 89.0 / F = 172.9513, ndf = 1, ddf = 199, p-value < 2.2e-16]

\section{Discussion}

\subsection{Des rencontres aux résultats probants}

Nous avons observé, à l'occasion de cette enquête, quelle forme a pris l'obligation de travailler ensemble imposée par l'institution lors d'un stage conçu en duo de stagiaires en formation professionnelle pour l'enseignement dans les classes primaires. Cette contrainte leur a permis d'expérimenter le «travailler ensemble », de s'y acculturer, d'y mettre du sens, d'y trouver, à ce qu'ils en disent, du bénéfice.

Les stagiaires ont clairement investi l'espace imposé des rencontres de coordination, de discussion, d'échanges. À quelques exceptions près, ils les ont trouvées productives, utiles, permettant d'effectuer des apprentissages dans le domaine professionnel. Travailler ensemble est cependant chronophage, cela est connu depuis longtemps. Contraints de le faire, les stagiaires ont utilisé tous les espaces possibles, dont celui des trajets en covoiturage, les cours dans lesquels ils n'interviennent pas ni n'observent activement, les récréations, la pause de midi au restaurant. Plusieurs rencontres ont eu lieu lors de jours fériés ou de congé. Mais cet aspect moins attractif du travailler ensemble ne les a pas arrêtés : nous avons pu constater que beaucoup de temps a été consacré à " travailler ensemble", au-delà de ce que requiert formellement l'institution (pour rappel: deux rencontres de formation et accompagnement du processus réflexif individuel par semaine d'une heure maximum et une rencontre quotidienne pour faire le point sur l'organisation didactique et pratique de la journée de classe). Si une réunion ou rencontre par jour pouvait être attendue, ce sont quasiment deux rencontres en moyenne qui ont été annoncées par les stagiaires. Ces rencontres ont eu lieu principalement entre les stagiaires et en présence de leur maitre de stage. Force est de constater que peu d'autres acteurs y ont pris part : nous nous serions attendue à voir intervenir un peu plus souvent quelques parents, directions, inspecteurs ou davantage de collègues du centre scolaire. Ce résultat pourrait indiquer que, à ce moment de début de formation, "travailler ensemble" tourne exclusivement autour de ce qui est très proche de la classe et quasiment uniquement avec les principaux intéressés. 

qui faire la classe dépend de leur capacité à la prendre puis à la tenir, les résultats de notre enquête montrent que les stagiaires de cette enquête ont comme préoccupation principale de la faire. La gestion de la discipline, les discussions relatives aux élèves en difficulté, les coordinations relatives aux travaux demandés par l'institut de formation à l'enseignement ne sont certes pas oubliées mais les préoccupations prioritaires ont trait aux contenus, à la gestion des cours et à leur organisation matérielle. Quelle explication à cela? On peut estimer que la gestion de la discipline, du prendre et surtout du tenir la classe, est prise en charge (implicitement, ce dont les stagiaires n'ont pas toujours conscience) par la seule présence du praticien-formateur - titulaire de la classe - puisque ce dernier est présent lorsque le stagiaire enseigne. Du coup, cette garantie de la tenue d'un cadre de travail adéquat ouvre l'espace à la discussion entre praticien-formateur et stagiaires à propos des aspects didactiques de l'enseignement (les contenus, les méthodes d'apprentissage...). En outre, le travail imposé par l'objectif prioritaire du stage (les différentes formes d'évaluation et leur articulation) renforce la nécessité d'y accorder une grande importance puisque la manière dont les novices évaluent les apprentissages des élèves au cours des séances qu'ils ont construites et qu'ils gèrent est observée tout au long du stage et évaluée en fin de stage.

\subsection{Les préoccupations partagées entre stagiaires : les sentiments et les émotions d'abord}

30 Les préoccupations des stagiaires, qui discutent significativement de certains objets plus entre eux qu'avec les autres acteurs, vont aussi dans le sens du souci qu'ils ont de « réussir » leur stage, selon les critères que vont évaluer le praticien-formateur et le superviseur HEP chacun de son côté et selon sa posture l'un de praticien, l'autre de référent académique. Ainsi, l'organisation matérielle de la journée ou des journées à venir et les contenus des cours, soit les aspects pratiques du stage qui vont permettre de faire au mieux la classe, ont été discutés avant tout avec le praticien-formateur. Par contre, les difficultés rencontrées et les réussites constatées pendant l'enseignement ont été surtout discutées avec un pair, avec son co-stagiaire, comme sont repris et commentés avec lui les entretiens de supervision menés soit avec le praticien-formateur ou avec le superviseur HEP.

Autre élément nouveau: la supervision HEP qui se trouve être un des sujets significativement plus discutés entre stagiaires. Lors de l'analyse descriptive, cet item n'était pas apparu comme prioritaire, alors que les items relatifs à l'organisation matérielle, aux difficultés et réussites rencontrées l'étaient. Nous interprétons ce résultat comme démontrant un souci propre aux stagiaires de satisfaire aux exigences d'un expert, que ce soit le praticien-formateur et ses exigences pratiques de gestion de la classe et de l'enseignement, ou l'un des superviseurs HEP et son regard didactique et théorique qui peut être pointu.

Parler entre pairs de sujets sensibles telles les difficultés, les réussites ou encore les entretiens de supervision avec le praticien-formateur ou avec le superviseur HEP, c'est peut-être parler d'abord de ses émotions, de ses peurs, de ses craintes, de soucis intimes que l'on ne dévoile pas à celui qui va évaluer. Avec ce dernier, les rencontres sont tournées vers la préparation concrète de l'enseignement, vers les analyses a priori et $a$ posteriori des leçons données et observées, et non vers les émotions ressenties.

Questions Vives, $n^{\circ} 21$ | 2014 
33 Mais, si l'on suit ce raisonnement, ce qui surprend, c'est que les rencontres des costagiaires entre eux - du moins à ce qu'ils en disent - sont perçues par eux comme étant plus formatrices (item: j'ai appris quelque chose) que celles qui sont menées avec le praticien-formateur (item: c'était une réunion d'échange d'informations ou d'impressions uniquement), lui dont le rôle est pourtant clairement défini dans une visée formative, lui qui est soigneusement préparé à accompagner, à coacher la construction réflexive des compétences et savoirs professionnels...

\section{En conclusion : travailler ensemble pour construire du développement professionnel en formation initiale : oui !}

Pour répondre à notre question initiale, à savoir comment les stagiaires investissent - s'ils investissent - mettent du sens, s'adaptent et s'approprient l'injonction travaillez ensemble ! que leur adresse l'institut de formation, comment ils organisent ce « travailler ensemble " imposé, quels objets y sont prioritairement discutés, nous pouvons dire que, bien que cette collaboration soit au départ subie, ils l'ont fait avec beaucoup de sérieux, entre eux ou avec leur praticien-formateur. Nous avons relevé que, prioritairement, ce sont les aspects didactiques et pratiques, l'organisation matérielle de la classe, la préparation des leçons données en duo ou individuellement, qui ont fait l'objet des discussions avec l'enseignant chevronné qu'est le praticien-formateur, réalisant là une forme de "compagnonnage réflexif » (Donnay \& Charlier, 2007). Les échanges que privilégient les stagiaires entre eux relèvent de préoccupations qui les concernent personnellement, qui sont peut-être difficiles à partager avec quelqu'un qui n'est pas un pair, qui appartiennent au domaine de la gestion des émotions, des sentiments.

Ces résultats positifs sont sans doute à mettre au bénéfice du «travailler ensemble » qui est de fait rendu obligatoire par la forme même du stage et ceci à plusieurs niveaux. Il y a certes les prescriptions, écrites et expliquées en réunion à tous les acteurs (stagiaires, praticiens formateurs, superviseurs), qui permettent à chacun de savoir ce qui est attendu de lui, de connaitre son rôle dans le stage et de tenir sa partition. Les stagiaires savent ce qu'ils ont à faire, comment et à l'aune de quels objectifs, de quelles exigences, ils seront évalués et verront - ou non - leur stage validé. Il y a ensuite un niveau du terrain, de la pratique qu'occupe le praticien-formateur, cet enseignant chevronné qui doit «tenir compte des particularités individuelles d'un stagiaire sans perdre de vue les exigences communes à tous » (Guillemette \& L'Hostie, 2011, p. 2) et est sans aucun doute soucieux de proposer au stagiaire - et nous sommes dans le cadre de la présente recherche en première année de formation - « un milieu authentique, certes, mais aussi 'protégé' et moins difficile (selon les phases de progression du stagiaire) que le milieu dans lequel intervient un enseignant professionnel au quotidien. La professionnalisation est, d'abord et avant tout, un processus évolutif, une progression » (Guillemette \& Lapointe, 2011, p. 26).

Dans la classe de stage, sachant précisément les objectifs qu'ils doivent atteindre et parce qu'ils ne sont pas seuls à devoir prendre et tenir la classe et ne sont pas vraiment responsables de la gestion de la discipline et du cadre de travail des élèves (ce qui sera par contre le cas pendant les stages de dernière année de formation où ils seront seuls garants de la qualité de ce cadre propice aux apprentissages), les stagiaires peuvent 
entrer rapidement, même en étant au début de la formation, dans les aspects didactiques de l'enseignement.

Il y a ensuite la relation personnelle développée avec le pair, ce collègue de stage avec qui on est contraint de travailler, de préparer, d'enseigner, de réfléchir, de réguler tant au niveau de l'enseignement qu'à celui des relations et avec qui, finalement, les stagiaires disent apprendre le plus. Ce résultat apparait finalement comme étant le plus intéressant de notre enquête. Car si les stagiaires placés en duo dans une classe reçoivent bel et bien quelques guides pour baliser les modalités de leur action commune, c'est bien dans les relations à l'intérieur du duo qu'ils rencontrent à la fois le plus d'autonomie mais aussi de contraintes. Travailler ensemble alors qu'on ne s'est pas choisi pour atteindre des objectifs communs, se fait ici dans une interdépendance positive puisque chacun doit prendre la responsabilité d'une partie de la tâche indispensable pour la réalisation du travail, doit développer une responsabilité collective et individuelle face à la tâche, est appelé à poser régulièrement une réflexion critique sur son fonctionnement et sur celui du duo, doit mener des interactions simultanées décidées par avance et coordonnées. Sont donc observés quelques-uns des facteurs de réussite d'un travail coopératif, facteurs identifiés notamment par Johnson et Johnson (voir à ce propos Rouiller \& Lehraus, 2008). Sont sans aucun doute aussi à l'œuvre des processus liés au conflit sociocognitif décrit par Vygostky, processus de confrontation à un problème, de prise de distance qui permet l'affirmation de sa propre conception à partir de ce que pense autrui au sujet de la même situation: sans vraiment l'expliciter aux acteurs, les responsables de l'organisation des stages à la HEP-VS ont tablé sur ces processus lorsqu'ils ont décidé, d'un point de vue formatif avant tout, de mettre les stagiaires en duo dans les classes. Les résultats de la présente recherche confortent la pertinence de ce choix.

Une autre limite observée pour ce stage concerne le champ des interlocuteurs avec lesquels un contact a été établi, champ restreint aux acteurs concernés directement par la classe, soit essentiellement le co-stagiaire et le praticien-formateur. Or, nous le savons, s'il l'a jamais été, l'enseignant est moins que jamais seul dans sa classe. Le slogan « de moi et ma classe à nous et notre école » parle d'acteurs de plus en plus divers et présents, qui interviennent, influencent voire interfèrent sur l'espace d'une classe (voir par ex. Tardif \& Levasseur, 2010, mais la tendance n'est pas propre à l'Amérique du Nord). De fait, c'est aussi le rôle de la formation que les résultats de cette recherche mettent en exergue : il reste encore deux ans à la disposition des stagiaires de la présente enquête pour s'ouvrir aux autres acteurs du monde scolaire. Différentes modalités de formation vont s'attacher à faire prendre conscience au futur enseignant de son rôle d'acteur social (Paquay, 1994) et à le développer. Pour remplir ce rôle, le jeune enseignant doit continuer à être attentif aux apprentissages des élèves (de prendre et tenir la classe, à la faire). Pour que le novice devienne un professionnel confirmé, en plus des compétences didactiques et en gestion de classe, il devra apprendre encore et toujours à se coordonner, à collaborer, à coopérer (Marcel et al., 2007) avec d'autres acteurs directement ou indirectement impliqués dans la vie scolaire. Il devra aussi s'intégrer dans les débats sociaux dans lesquels l'école est entrainée, dans un mouvement centrifuge dans lequel la classe, de noyau de départ, devient un des éléments constitutifs de la constellation sociale dans laquelle évolue l'enseignant, mais assurément pas le seul et unique. 


\section{BIBLIOGRAPHIE}

Amigues, R. (2003). Pour une approche ergonomique de l'activité enseignante. Skôlé, hors série, 1, $5-16$.

Barrère, A. (2002). Les enseignants au travail. Routines incertaines. Paris : L'Harmattan.

Buysse, A. (2009). Médiations, régulations et développement : une base pour penser la formation des enseignants. Revue suisse des sciences de l'éducation, 3/2009.

Buysse, A. (2011). Une modélisation des régulations et de la médiation dans la construction des savoirs professionnels des enseignants. In P. Maubant \& S. Martineau (Éds.), Fondements des pratiques professionnelles des enseignants (pp. 243-284). Ottawa : Presses de l'Université d'Ottawa.

Lumley T. (2010). Complex Surveys: A Guide to Analysis Using R. Hoboken : Wiley.

Lessard, C. (2010). Le travail enseignant, entre les effets structurants des politiques et l'activité en classe, la part des sujets. In Yvon, F. \& Saussez, F. (Éds.), Analyser l'activité enseignant. Des outils méthodologiques et théoriques pour l'intervention et la formation. Québec : Presses de l'Université Laval.

Letor, C. (2009). Comment travailler en équipe au sein des établissements scolaires ? Bruxelles : De Boeck.

Corriveau, L., Letor, C., Périsset, D., \& Savoie, L. (Éds.). (2010). Travailler ensemble : dans les établissements scolaires et de formation : processus, stratégies et paradoxes. Apprentissages individuels, compétences collectives et développement professionnel en organisation. Bruxelles : de Boeck Université.

Donnay, J., \& Charlier, E. (2007). Apprendre par l'analyse des pratiques : initiation au compagnonnage réflexif ( 2 éd.). Sherbrooke : CRP.

Dupriez, V. (2007). Quelles relations entre les formes organisationnelles et les formes de l'action éducative au sein des établissements? In J.-F. Marcel, V. Dupriez, D. Périsset \& M. Tardif (Éds.), Coordonner, collaborer, coopérer: de nouvelles pratiques enseignantes (pp. 21-34). Bruxelles : de Boeck Universités.

Guillemette, F., \& L'Hostie, M. (Éds.). (2011). Favoriser la progression des stagiaires en enseignement. Québec : Presses de l'Université.

Guillemette, F., \& Lapointe, J.-R. (2011). L'autoformation du stagiaire. In F. Guillemette \& M. L'Hostie (Éds.), Favoriser la progression des stagiaires en enseignement (pp. 5-33). Québec : Presses de l'Université.

Lumley T. (2010), Complex Surveys : A Guide to Analysis Using R, Hoboken : Wiley.

Marcel, J.-F., Dupriez, V., Périsset D., \& Tardif, M. (Éds.). (2007). Coordonner, collaborer, coopérer : de nouvelles pratiques enseignantes. Bruxelles : de Boeck Universités.

Marcel, J.-F., \& Garcia, A. (2010). Pratiques enseignantes de travail partagé et apprentissages professionnels. In L. Corriveau, C. Letor, D. Périsset \& L. Savoie (Éds.), Travailler ensemble : dans les établissements scolaires et de formation : processus, stratégies et paradoxes. Apprentissages individuels, compétences collectives et développement professionnel en organisation (pp. 15-30). Bruxelles : de Boeck Université. 
Paquay, L. (1994). Vers un référentiel des compétences professionnelles de l'enseignant? Six paradigmes. Recherche et Formation, 16, 7-38.

Périsset, D. (2009). Former à l'accompagnement en stage et à la supervision pédagogique : enjeux et défis. Les propositions de la Haute école pédagogique du Valais (Suisse). In J.-F. Desbiens, C. Borges \& C. Spallanzani (Éds.), La supervision pédagogique en enseignement de l'éducation physique. Éducation et francophonie, XXXVII, 1, 50-67. En ligne : http://www.acelf.ca/c/revue/pdf/ EF37-1-051_PERISSET.pdf (consulté le 26/08/2014)

Périsset, D. (2010). L'acculturation au travailler ensemble : un défi posé à l'alternance en formation initiale. In L. Corriveau, C. Letor, D. Périsset \& L. Savoie (Éds.), Travailler ensemble : dans les établissements scolaires et de formation : processus, stratégies et paradoxes. Apprentissages individuels, compétences collectives et développement professionnel en organisation (pp. 137-152). Bruxelles : de Boeck Université.

Périsset, D. (2012). L'évaluation des candidat-es à l'enseignement primaire en fin de formation : sens de la mesure, petit tour d'horizon helvétique. In R. Etienne \& L. Clavier, Évaluation dans la formation des enseignants (pp. 145-164). Paris : L'Harmattan.

Périsset, D., \& Buysse, A. (2008). La pratique réflexive entre intentions et situations de formation. In L. Mottier Lopez, Y.-E. Dizerens, G. Marcoux \& A. Perréard Vité (Éds.), Entre la régulation des apprentissages et le pilotage des systèmes : évaluations en tension. Actes du $20^{\mathrm{e}}$ colloque ADMEEEurope, Université de Genève, Suisse.

$R$ Core Team (2013). R: A language and environment for statistical computing. $R$ Foundation for Statistical Computing. Vienna, Austria. URL http://www.R-project.org/ (consulté le 29/08/2014).

Rouiller, Y., \& Lehraus, K. (Éds.). (2008). Vers des apprentissages coopératifs : rencontres et perspectives. Berne : Peter Lang, Exploration.

Saujat, F. (2004). Comment les enseignants débutants entrent dans le métier. Formation et pratiques d'enseignement en question, 1, 97-116. En ligne : http://www.revuedeshep.ch/site-fpeq/ Site_FPEQ/1_files/2005-1-Sauja.pdf (consulté le 29/08/2014).

Tardif, M., \& Levasseur, L. (2010). La division du travail éducatif. Une perspective nord-américaine. Paris : PUF.

\section{NOTES}

1. Il y a un stage de 3 à 5 semaines par semestre d'études, pour un total d'une trentaine de semaines de stages répartis sur 6 semestres. Pour une description des principes de cette formation en alternance, voir Périsset, 2009, 2012.

2. Soit : les stagiaires, les maitres de stages appelés praticien-formateur (PF), les responsables des cours dispensés en institution pendant le semestre concerné, les superviseurs HEP, la commission HEP responsable de l'organisation des stages, le formateur responsable de l'organisation du stage.

3. À relever que lors de ce stage dit stage 201, l'institution exige formellement la tenue de deux entretiens de formation hebdomadaires entre stagiaires et maitre de stage, d'une heure au maximum, au cours desquels la progression vers les objectifs de formation sont discutés. D'autres rencontres, dites de planification, doivent avoir régulièrement lieu, selon les besoins et hors des jours pendant lesquels sont planifiés les entretiens de formation. Ces rencontres, axées sur les questions organisationnelles, doivent durer au maximum une heure également. 
4. Nous remercions vivement le professeur Paul Ruppen, de la HEP-VS, pour les traitements statistiques qu'il a réalisés pour cette enquête.

5. Référence pour R : voir R Core Team (2013).

6. Pour lire les tableaux : les effectifs renvoient aux fréquences observées et les effectifs théoriques aux fréquences auxquelles on peut s'attendre si les deux variables sont indépendantes. Le résidu note la différence entre effectif et effectif théorique. L'item " choisi » renvoie au nombre de fois où il a été coché (choisi) par les étudiants, « non choisi » au nombre de fois où les étudiants l'ont ignoré (non choisi).

7. Du point de vue statistique, les acteurs ont été traités comme strates, chaque strate étant constituée par les questionnaires d'un acteur. Les valeurs [p] ont été calculées avec une méthode bootstrap (200 tours) à l'aide du R-paquet Survey (commande : svychisq(variable1+variable2, boot_design). Pour une explication de la méthode, voir Lumley (2010). Pour le logiciel $R$ voir $R$ Core Team (2013).

\section{RÉSUMÉS}

Cet article analyse la manière dont des stagiaires en formation à l'enseignement au degré primaire en Suisse investissent l'espace collaboratif consubstantiel d'un stage en duo. Un questionnaire a été soumis à une cohorte de 45 jeunes en première année de formation. 1285 rencontres ont été recensées. Il s'est avéré que ces moments ont été fortement investis par les stagiaires qui les ont trouvés utiles. Les objets discutés montrent principalement le souci de «faire» la classe, et pas seulement la «prendre » et la «tenir». Dans ce sens, l'obligation de «travailler ensemble» est formatrice. Les stagiaires ont cependant eu des sujets de discussion différents selon leur interlocuteur, maitre de stage ou costagiaire. Enfin, les acteurs présents lors des rencontres formatives sont dans une dynamique de "moi et ma classe » et non "nous et notre école, dans son espace social ", dynamique qui devra être travaillée en stage au cours de deux ans de formation encore à venir.

This article analyzes how students in teacher education at the primary level in Switzerland invest the collaborative consubstantial space of an internship duo. A questionnaire was administered to a cohort of 45 first year trainees. 1285 meetings were recorded. It turned out that these moments were strongly invested by students who found them useful. The items discussed mainly show concern for teaching, not just classroom management. In this sense, the obligation to "work together" is formative. Trainees, however, had different discussion topics according to their discussion partner (supervisor or co-trainee). Finally, the actors present during the formative meetings are in a "me and my class" dynamic, not "us and our school, in its social space". This dynamic will have to be worked on during the internships, throughout the two coming years of training.

\section{INDEX}

Mots-clés : travail collaboratif, travailler ensemble, formation à l'enseignement, stage, novices Keywords : collaborative work, work together, teacher education, internship, novices 


\section{AUTEUR}

DANIĖLE PÉRISSET

Haute école pédagogique du Valais et Université de Genève (Suisse) 